\title{
Multiple Signalling Transduction Mechanisms Differentially Coupled to Somatostatin Receptor Subtypes: A Current View
}

\author{
Davide Cervia ${ }^{*}$, , Caroline Nunn $^{2}$ and Paola Bagnoli ${ }^{1}$ \\ ${ }^{1}$ Dipartimento di Fisiologia e Biochimica “G. Moruzzi”, Università di Pisa, 56127 Pisa, Italy \\ ${ }^{2}$ Department of Physiology and Pharmacology, University of Western Ontario, N6A 5C1 London, Ontario, \\ Canada
}

\begin{abstract}
Somatostatin (SRIF) is a cyclic peptide widely distributed throughout the body with important physiological effects (mostly inhibitory) on several organ systems. SRIF may act as a neurohormone, neurotransmitter, neuromodulator or as a local factor, and exhibits potent antiproliferative activity. SRIF effects have formed the basis for the clinical use of SRIF analogues in the treatment of endocrine tumours, acromegaly and gastrointestinal disorders. Several data suggest that SRIF may also be a therapeutic target in a number of different diseases.

The binding of SRIF to its five G-protein coupled receptors leads to modulation of multiple transduction pathways, including adenylyl cyclase, guanylyl cyclase, phospholipase $\mathrm{C}, \mathrm{K}^{+}$and $\mathrm{Ca}^{2+}$ channels, phospholipase $\mathrm{A}_{2}$, nitric oxide, $\mathrm{Na}^{+} / \mathrm{H}^{+}$exchanger, protein phosphatases and MAP kinases. The diversity of the transduction pathways reflects the pleiotropic actions of SRIF. However, our current understanding depicts a rather complicated picture and conflicting results have also been reported. Data are mostly based on in vitro experiments, and parallels with the real in vivo conditions are not so obvious. Due to the clinical relevance of the SRIF system, the elucidation of the intracellular role of endogenous SRIF receptors may offer new therapeutic perspectives. These will enable development of specific pharmacological signalling modulators which can be incorporated into the therapeutic arsenal.

The present review represents a detailed and exhaustive summary which covers the latest advances in the transduction pathways of SRIF receptors.
\end{abstract}

Keywords: Endogenous receptor, recombinant system, G-protein, cyclic nucleotide, enzyme, nitric oxide, phospatase, kinase.

\section{INTRODUCTION}

\section{The Peptide Somatostatin}

Somatostatin or SRIF (somatotropin release inhibiting factor) is one of the longest known neuropeptides. It was first discovered in 1968 in hypothalamic extracts as a low molecular weight peptide which was shown to inhibit growth hormone $(\mathrm{GH})$ secretion from cultured anterior pituitary [1]. In 1973, the same peptide, purified from bovine pituitary, was also found to potently inhibit GH release and was named somatostatin [2]. In mammals, SRIF is produced from a single gene which encodes preprosomatostatin (116 amino acids). This is processed to prosomatostatin (96 amino acids) which is further cleaved to produce two bioactive products: SRIF-14, which was the fourteen amino acid peptide originally discovered, and SRIF-28, which has an N-terminal extension of fourteen additional amino acids [3].

SRIF is present in mammals and many non-mammalian vertebrate classes including birds, amphibians, reptiles and fish [4-8]. In general, it is involved in the regulation of the functions of several organ systems including, for instance, the central nervous system, the pituitary gland, the

\footnotetext{
*Address correspondence to this author at the Dipartimento di Fisiologia e Biochimica "G. Moruzzi", Università di Pisa, 56127 Pisa, Italy; Tel: +39050-2213459; Fax: +39-050-2213527; E-mail: d.cervia@dfb.unipi.it
}

gastrointestinal tract, the endocrine and exocrine pancreas, several components of the immune system, and vessel walls $[4,9]$. Besides inhibiting GH secretion, SRIF has been found to negatively regulate the secretion of many other hormones including thyrotropin, corticotrophin releasing hormone, adrenocorticotrophic hormone, insulin, glucagon, secretin and vasoactive intestinal peptide hormone [4, 9]. SRIF also inhibits a variety of other physiological functions such as gastrointestinal motility, gastric acid production, pancreatic enzyme secretion, bile secretion and colonic fluid secretion. In addition, SRIF exhibits potent antiproliferative activity (cell-growth arrest and/or apoptosis) on both cultured cells and tumours in experimental animals [3,9]. Finally, it acts in the central nervous system with effects on motor, sensory, cognitive and autonomic functions $[10,11]$. In these different activities, SRIF acts as a neurohormone, a neurotransmitter, a neuromodulator or as a local factor acting via autocrine or paracrine mechanisms.

Pathologically, SRIF has been linked to a number of diseases including acromegaly, gastrointestinal disorders, cancers, rheumatoid arthritis, diabetic retinopathy and cystoid macular oedema $[9,12,13]$. Since it is expressed and active in the central nervous system it is not surprising that SRIF is also involved in various psychiatric or neurological diseases including Alzheimer's disease [14, 15], Huntington's disease [16, 17], Parkinson's disease [18], HIV encephalitis [19], epilepsy [20], depression, schizophrenia [21] and dementia [22]. 